\title{
Summary of Spacecraft Charging
}

\author{
Maosong Yang ${ }^{a, *}$, Yongwei Sun, Zhicheng Meng ${ }^{b}$ and Lidong Zhou ${ }^{c}$ \\ Ordnance Engineering College, Shijiazhuang 050003, China; \\ a1084610202@qq.com, b1816500194@qq.com, ${ }^{7} 758668793 @ q q . c o m$
}

Keywords: spacecraft; surface charged; medium deep charge.

\begin{abstract}
With the continuous development of science and technology, the convenience spacecraft brought to us has been everywhere. During the orbit operation, the spacecraft interacts with the complex space environment, which makes the surface and internal materials appear charging and discharging phenomenon, and thus may lead to shortened its life or cannot work properly, or even scrapped and so on. The surface charge and the medium charge of the spacecraft during orbital operation are discussed in depth. It has certain reference value for the system to study the spacecraft charged and the protection design of spacecraft charge and discharge.
\end{abstract}

\section{Introduction}

Most of the orbital environment around the earth is in the plasma state. The space is mainly composed of solar wind plasma, magnetospheric plasma and ionospheric plasma. They are complex effects of solar electromagnetic radiation, particle irradiation interact with the Earth's magnetic field and the Earth's thermal residual atmosphere. Therefore, the spacecraft during orbital operation will be subject to space plasma, high-energy electrons and solar radiation and other environmental effects. Solar photon emission can also cause the occurrence of charging. In addition, the radioactivity parameters such as the volume resistivity, surface resistivity, and secondary electron emission coefficient of the spacecraft material, as well as the surface charge condition and the material structure of the material, will have an important effect on the spacecraft charging process. Electrostatic discharge can cause the breakdown of the surface material of the spacecraft, the decline of the performance of the solar cell array and the resulting electromagnetic pulse interference will cause the star on the sensitive electronic equipment / system misuse or damage, thus affecting the safe operation of the spacecraft in orbit. In order to minimize the impact of charging, in-depth understanding of spacecraft charged is particularly important.

\section{The Charging of Spacecraft Surfaces}

Spacecraft running in space interact with the surrounding plasma, magnetic field and solar radiation and other environmental factors, which result in the accumulation of charge on the surface of the spacecraft. The phenomenon that space between the spacecraft and space between different parts of the spacecraft charge with different potential is known as the spacecraft charged, which is also known as spacecraft surface charge.

The effect of surface charge on spacecraft was found in the 1970s. The United States found more than 50 satellites in the geosynchronous orbit have many anomalies. And these anomalies seriously disrupted the normal work of the satellite, which attracted the attention of the design department. They organized a total of 19 members to make a system analysis of the four series Satellite accidents occurred found that there are three main types of problems: electromagnetic interference, power and sensitive device damage and surface material degradation. The study found that these phenomena occurred in the geomagnetic disturbance, and most of the place between 00 to 06 points. Through the analysis of the results of the onboard instrument, with a large number of ground simulation test, it was known that these accidents ware due to geomagnetic sub-storm from the magnetic tail into a group of high-temperature plasma (energy about $20 \mathrm{keV}$ ) which caused spacecraft charged. Therefore, 
the surface charge research as a guarantee of geostationary orbit satellites normal work was put forward.

The surface charge of the spacecraft is divided into two types: absolute charge and unequal charge. Absolute charging refers to the potential of the spacecraft structure relative to the surrounding space plasma. Unequal charge is the unequal potential changes between the different surfaces of the spacecraft. Among them, unequal charge is the main cause of space discharge on the spacecraft. The reason is that the surface of the spacecraft is covered by a variety of materials, and the secondary electron emission coefficient and the backscattering coefficient of different materials are different. So they have different equilibrium potential, and the potential difference between adjacent surfaces lead to unequal charge. The surface of the spacecraft and the back surface of the spacecraft will also cause unequal amount of electricity which is due to photoelectron emission. The difference between the "ground" potential of the spacecraft and the average surface of the spacecraft is related, and the difference between the surface dielectric material potential and the underlying conductor potential is different from the charge of the spacecraft.

Since the continuous flow of current can not be formed in the space, the charged particles in the plasma can charge the spacecraft to several times the potential of the electron energy. Through the surface charging process, the spacecraft running in the plasma environment and the surrounding plasma could achieve electrical balance, that is, the entire space system and independent insulation surface current is zero. The equilibrium condition determines the surface voltage of the spacecraft relative to the surrounding plasma. The surface current of the spacecraft is mainly composed of two parts: one is the primary current formed by the collection of electrons and ions in the space plasma, another is related to the surface material properties such as secondary electron emission, backscattered electrons and Photoelectron current and so on.

Spacecraft surface charging is a more complex process, the surface charge potential is related to space environment, spacecraft structure, size and surface material performances do so on. In addition, the parameters that affect the surface of the spacecraft are: spatial electron beam density, spatial ion beam current density, material photocurrent density, electron-generated material secondary electron emission coefficient, material backscattering coefficient, ion generated material secondary the electron emission coefficient, the bulk resistance of the material, and the surface resistance of the material. In addition to the above factors, the structure of the spacecraft (including the material area, size and layout structure, etc.) and the working conditions of components (power status and grounding conditions) and other factors will also affect the surface of the spacecraft material. Therefore, all of the above factors need to be taken into account in the design of spacecraft protection methods.

In recent years, there have been some new developments in the theory of surface charge of spacecraft. For example, the asymmetry of space environment parameters, tail charge, overcharging, etc., which have provided great help for the accuracy of surface charge distribution analysis. Among them, in the low Earth orbit (LEO), the speed of the spacecraft is greater than the ion velocity and much smaller than the electron velocity, and the ion "vacuum" zone formed at the tail of the spacecraft is called the tail zone. In the tail region, due to the lack of ion neutralization, the spacecraft surface is prone to a higher negative potential. Especially for polar orbiters, when the auroral electrons are injected into the tail of the spacecraft, the tail surface of the spacecraft can form extremely severe uncharged quantities in a short time. The charge effect of the tail zone is a surface charge effect, and its charge degree is directly related to the size of the spacecraft. Foreign studies have shown that the diameter of $10 \mathrm{~m}$ satellite satellites (or antenna and other large payload) tail area charge potential up to tens of thousands of volts. The low-orbit reconnaissance satellites running on the pole are usually have large antennas. When they pass through the polar regions, the surface will have a higher potential. Due to the large charge capacitance of the large structure, the energy generated by the discharge is huge. Damage is also more serious.

\section{Medium Deep Charge}

Large amounts of high-energy electrons are injected into the geosynchronous orbit or the sun's orbit in a strong disturbance environment such as solar flare outbreaks, coronal mass ejections, 
geomagnetic storms, or geomagnetic subbursts, resulting in a significant increase in electron flux greater than $1 \mathrm{MeV}$. These electrons can directly penetrate the surface of the satellite surface, the satellite structure and equipment shell. In the satellite internal circuit board, wire insulation layer and other insulating media deposition, they can result in the accumulation of charge and cause the media deep charge. It is also known as medium deep charge or body charged, which is now considered to be the main reasons caused high-orbit satellites in orbit abnormalities.

High energy particle irradiation mainly comes from galactic cosmic radiation, geomagnetic capture radiation, solar proton events and artificial radiation. The main energy is $1 \sim 40 \mathrm{MeV}$. Galaxy cosmic radiation is the charged particle flow from the outside solar system. Galaxy cosmic rays are essentially homogeneous. Because of the high energy of the particles, it is difficult to shield it. But its flux is very low, and the measurement is generally not more than a few millrades. The geomagnetic capture radiation zone refers to the area of high-energy charged particles captured by the geomagnetic field in the Earth's magnetosphere, sometimes referred to as a geomagnetic capture zone. Since the Earth's radiation was first discovered by American scholar Van Allen, it was also called the Van Allen radiation zone. The solar proton event, also known as the solar particle event, refers to a large number of high-energy proton emissions accompanied by solar flares. The proton energy is $10 \sim 1000 \mathrm{MeV}$. In addition to protons, there will be a small amount of heavy nuclei and $\alpha$ particles. When a solar proton event occurs, magnetic storms, ionospheric disturbances, and solar X-ray bursts may also occur.

For media, high-energy charged particles $(\mathrm{MeV})$ penetrating into the medium will remain in the medium. The reason is that the conductivity of the dielectric material is low. In the energy range of several tens of $\mathrm{MeV}$, the penetration depth of electrons is much deeper than that of ions, and a negative charge region is formed deeper than the ion layer at a certain depth. For a few days, months or even years, spacecrafts are exposed to high-energy particle environment, and the material within the electronic accumulation may be established within the dielectric high electric field. When the electric field inside the medium exceeds the breakdown threshold of the dielectric material, the discharge will occur. The generated electromagnetic pulse will interfere with or even destroy the normal work of the internal electronic system of the spacecraft.

The surface of the spacecraft is usually composed of a dielectric material (optical coating, solar cell cover or exposed solitary wiring) that has a conductive substrate and material that is connected to the space of the spacecraft. These media are prone to internal charging. The most important is that the space high-energy charged particles have a "penetrating power" that can pass through the surface of the spacecraft and deposit it into the medium to produce internal charge.

The high energy $(\mathrm{MeV})$ electrons and ions impinging on the surface material penetrate different depths, and the penetration depth is related to particle energy, particle type and material properties. At the energy level of $\mathrm{keV}$, electrons and ions can only penetrate into shallow depths near the surface, and their penetration depth is not significantly different. In the order of energy for $\mathrm{MeV}$, electrons penetrate deeper than ions, and their penetration depth differences will become very noticeable. At an energy level of $100 \mathrm{MeV}$ or higher, the penetration depth of ions is greater than that of electrons, which may trigger meson products and nuclear reactions. For the medium charged with spacecraft in the Earth's radiation belt, we focus on the electron and proton fluxes in the energy range of 0.01 to 20 $\mathrm{MeV}$, and the flux of higher energy electrons and protons is very small. Electrons and ions can penetrate to different depths, which are related to energy. Below $0.01 \mathrm{MeV}$, the range of electrons and protons is approximately equal; above $100 \mathrm{MeV}$, proton penetration is deeper than electrons. The electrons and protons penetrating into different depths may remain in the material for several days or months, which is related to the electrical conductivity of the material. Accumulation and gradual increase in the internal charge will eventually establish a strong electric field (Gauss theorem), and this feature is the cause of the media deep charge. 


\section{Acknowledgments}

Many people have made invaluable contributions to my study. First, I would like to express my deepest gratitude to my advisor, Dr. Yongwei Sun. Without his support and guidance throughout the study, I would not get improvement in my study. His continued support led me to the right way.

I would also like to extend my appreciation to my senior fellow apprentice: Lidong Zhou for his advice during my study. I would also like to extend my deepest gratitude to my family. Without their encouragement, I would not have a chance to be here.

My thesis comes from National Natural Science Foundation of China: NO. 51577190.

\section{References}

[1] Prebola J L, Bertrand W T, Crider D H, et al. Development of a combined space environment test facility at AEDC. Aerospace Testing, 2006.

[2] Shu T L. Fundamentals of spacecraft charging. New Jersey: Princeton University Press,2012.

[3] Henery B G, Albert C W. Guide to mitiating spacecraft charging effects. New Jersey: John Wilery \& Sons, 2012.

[4] Finckenor M M, Kamenetzky R R, Vaughn J A, et al. Space environmental effects testing in support of the international space station. 38th AIAA Aerospace Sciences Meeting and Exhibit, 2000, 01.

[5] Liu Shanghe,Wei Guanghui,Liu Zhicheng,et al. Electrostatic Theory and Protection. Beijing:Ordnance Industry Press, 1999.

[6] Huang Bencheng,Tong Jingyu.Space Environmental Engineering,Bingjing.China Science and Technology Press,2010. 